\title{
Ethik und Mythos der Hämotherapie*
}

\author{
Heinz-Josef Fabry \\ Alttestamentliches Seminar, Katholisch-Theologische Fakultät, Universität Bonn, Deutschland
}

\section{Schlüsselwörter}

Ethik · Hämotherapie

\section{Zusammenfassung}

In der vorliegenden Arbeit wird die etwas ungünstige Wahrnehmung der Hämotherapie in der populären Beurteilung diskutiert. Nach einer kurzen Einführung in die Geschichte der indogermanischen (und semitischen) Linguistik werden die Hauptreferenzen im Alten Testament und in den mythologischen Werken des antiken Nahen Ostens - Griechenland, Altes Israel, Mesopotamien und Ägypten - analysiert. Das zentrale Mysterium der christlichen Eucharistie ist das Glaubensbekenntnis " ... dies ist mein Blut", das auf eine wundervolle christliche Hämotherapie hinweist, da das Vergießen des Blutes von Jesus Christus ein zentrales Element in der Erlangung der Erlösung und des ewigen Lebens ist. Die Verwendung von Blut in antiken Ritualen weist wichtige ethische Implikationen auf. Die Untersuchungen werden in 8 Thesen zusammengefasst.

Die vorliegende Arbeit versucht, die mythischen und ethischen Grundlagen des heilenden Umganges mit Blut zu ergründen. Wohl allen in unserer Gesellschaft ist die fundamentale Bedeutung des Organs «Blut» für das Leben und die nötige Sorgfalt mit ihm unmittelbar einleuchtend. Das Wissen darum ist so tief verankert, dass man darüber die hoch spezialisierten Wissenschaftlerinnen und Wissenschaftler, die mit diesem Blut heilend umgehen, kaum noch wahrnimmt. Ein Patient, dessen Zustand erst durch eine Bluttransfusion

*Dedicated to Prof. Dr. Peter Hanfland, Bonn, on the occasion of his 65th birthday.

\section{Key Words \\ Ethic · Haemotherapy}

\section{Summary \\ Ethic and Myth of Haemotherapy}

In this contribution the misjudgement of haemotherapy in popular estimation is discussed. After a short investigation of the history of indogerman (and semitic) linguistics the main references in Old Testament and mythological works of the Ancient Near East, i.e. Greece, Old Israel, Mesopotamia and Egypt, are analysed. The central mystery of Christian eucharist is the creed: '... this is my blood', that indicates a wonderful Christian 'haemotherapy', because the shedding of the blood of Jesus Christ is the central way to salvation and eternal life. The use of blood in antique rituals shows important ethical implications. The investigations done by the author are condensed into 8 theses.

entscheidend gebessert werden konnte, bedankt sich in der Regel bei seinem behandelnden Arzt, nicht beim Transfusionsmediziner, von dessen Existenz er nicht einmal weiß. Wie das Wasser aus dem Hahn, wie die Milch aus der Tüte, so kommt das Blut aus der Konserve. Dass dahinter ein riesiger, technisch aufwändiger und ökonomisch teurer Apparat mit hoch qualifiziertem Personal steht, wird dem Patienten in der Regel nicht bewusst.

Auch die gegenwärtig verhandelten medizinisch-ethischen und biomedizinisch-ethischen Fragestellungen sehen im Blick auf die Hämotherapie etwa im Gegensatz zur Samenspende keinerlei Beratungsbedarf: «Man kann ... eine homologe Insemination nicht einfach mit einer Bluttransfusion oder einer Netzhautübertragung vergleichen» [1]. Ist Hämotherapie tat-

Prof. Dr. Heinz-Josef Fabry

Alttestamentliches Seminar, Katholisch-Theolooische Fakultät

Rheinische Friedrich-Wilhelms-Universität

Regina-Pacis-Weg 1a, 53111 Bonn, Deutschland

Tel. +49 228-737886, Fax -284173

E-mail fabry@uni-bonn.de 
sächlich ethisch ohne Befund? Es wird sich zeigen, dass das von Hans Jonas propagierte «Prinzip Verantwortung» gerade im Umgang mit Blut die entscheidende Rolle spielt. Die Frage nach der Verantwortung ist aber die ethische Frage schlechthin! Es ist deshalb erneut der Nachweis zu führen, dass die ausbleibende Wahrnehmung und Wertschätzung des Blutes und des Umganges mit ihm sich konträr verhält zu seiner Bedeutung in der gesamten Menschheitsgeschichte.

Nach einem kurzen Einblick in die Sprachgeschichte möchte ich Sie in das Alte Testament und seine altorientalische Kulturgeschichte hineinnehmen. Anschließend ist einzugehen auf das Geheimnis der christlichen Eucharistie, das sich in dem Bekenntnis «... das ist mein Blut» konzentriert. Des Weiteren befassen wir uns mit der Mythenwelt Griechenlands, Mesopotamiens und Ägyptens. Im Blick auf die Verwendung von Blut in antiken Ritualen geht es darum, die ethischen Implikate aufzuspüren, die dann als Ausblick noch einmal zusammengefasst werden. Für die hier nicht zu behandelnden Bereiche (arabischer Galenismus, Mittelalter, mystisch geprägtes Wallfahrtswesen zu Stätten mit Heilig-Blut-Reliquien z.B. in Brügge und Walldürn, Vampir-Frage, Blut-und-Boden-Thematik usw.) sei auf Gadebusch Bondio [2] verwiesen.

\section{Die Terminologie «Blut» in unserem Sprachkreis}

\section{Im indogermanischen Bereich}

Die Herkunft dieses Wortes bietet bereits die erste Überraschung: Die ursprünglichen indoeuropäischen Bezeichnungen éar und krew sind allesamt sprachgeschichtlich ersetzt worden! So entstammt unser Wort «Blut» dem germanischen $b l \bar{o} d a$, das wiederum aus dem indogermanischen Verb bhel, «schwellen, strotzen, [auf]blasen, quellen, sprudeln» [3] entwickelt wurde. Es will also umschreiben: «Hervorquellendes Blut wird danach metaphorisch als «etwas üppig Gesprossenes» vorgestellt» [4]. Auch das lateinische sanguis und das griechische haima, woraus sich ja die «Hämatologie» herleitet, sind solche Ersatzwörter. Das Überraschende liegt also darin, dass alle unsere Wörter für «Blut» regional gebildete Ersatzwörter [5] sind, die das eigentlich Gemeinte aus Tabugründen umschreiben: man vermeidet die Nennung der eigentlichen Sache aus Scheu, «den «Namen» der gefürchteten und unheimlichen Sache auszusprechen, um die in ihr latente Kraft nicht zu wecken» [6]. Aus dieser Wortwurzel hat sich in der Folgezeit ein weites Wortfeld entwickelt, aus dem ich nur das beliebte Wort «blutjung» herausgreifen möchte, das eben nichts mit Blut zu tun hat, sondern auf das etymologisch gleiche «blühen, Blüte» zurückgeht. Aber auch diese «Ersatzwurzel» wurde in einigen Sprachbereichen tabuisiert: so wurde das germanische, altnordische und althochdeutsche Verb blo tan in der Bedeutung «jemanden mit Opfer ehren» schon relativ früh das dem lateinischen «offerre» «darbieten, darbringen» entlehnte «opfern» ersetzt und geriet dann selbst in Vergessenheit.
1. These: Der sprachliche Befund - die Vermeidung des Urwortes - signalisiert eine ehrfurchtsvolle Distanz zum Gemeinten.

\section{Im semitischen Bereich}

Die semitische Sprachfamilie denkt hier anders. «Blut», hebräisch und gemeinsemitisch dam [7] (eine Verbindung zum Adjektiv 'adom «rot» und 'adam «Mensch» lässt sich vermuten, aber nicht beweisen), ist wie ' $a b$ «Vater» und 'em «Mutter» eine Primärbezeichnung [8], im Gegensatz zu dem sekundären Rang des Wortes «Blut» in den indogermanischen Sprachen. Interessanterweise kennt der Hebräer eine Pluralbildung damîm [Koch 9], Ausdruck einer Affektionswertigkeit für das gewaltsam vergossene Blut, das auf dem Mörder lastet. Wie weit entfernt der Hebräer noch von der Entdeckung des Blutkreislaufes war, ersieht man daraus, dass er das Blut nie mit dem Herzen (leb) zusammen nennt, wohl aber mit der «Gurgel»(nжркеš), die als Sitz der physischen Lebenskraft verstanden wurde.

\section{Das Blut im Alten Testament und seine mythologischen Bezüge}

\section{Die Erzählung von Kain und Abel (Genesis 4)}

Schon die erste Blutnennung im Alten Testament ist zugleich die Schilderung einer «Bluttat», des ersten Brudermordes in der Menschheitsgeschichte. Diese Erzählung wird von den biblischen Verfassern im Rahmen der Anfangskapitel der Bibel erzählt, in denen es um die Grundordnung des menschlichen Zusammenlebens geht.

Die Brüder Kain und Abel bringen Gott ein Opfer dar, der Bauer Kain Getreide, der Hirte Abel ein Lamm. Gott akzeptiert das vegetabilische Opfer des Kain nicht, das geschlachtete Opfer des Abel nimmt er dagegen an und löst damit den ersten tödlichen Konflikt der Menschheit aus. Akzeptanz des einen und Verwerfung des anderen Opfers wird hier zwar als Sache Gottes dargestellt, was fälschlich zu einer Verdunkelung und Brutalisierung des Gottesbildes führte. Es geht der Erzählung aber gar nicht darum darzustellen, was die Opfer bei Gott erreichen, sondern wie sie auf die Opferspender selbst zurückwirken: das Blutopfer beruhigt, besänftigt, versöhnt den «Opferer» Abel, das vegetabilische Opfer, das ohne Blutvergießen auskommt, führt dagegen zur Eskalation bei Kain. Dahinter kann man eine mittelbare antike Hämotherapie erkennen: mit dem Blutvergießen des Opfertiers ist offensichtlich eine Kanalisierung menschlicher Gewaltpotentiale verbunden, die ansonsten unweigerlich unkontrolliert bleiben und Schaden anrichten würden [10].

\section{Mythen der ägyptischen Göttin Hathor}

Interessanterweise finden wir ähnliche Vorstellungen in Mythen der ägyptischen Göttin Hathor. Aus dem ägyptischen «Buch von der Himmelskuh» aus dem Neuen Reich ist ein Text bekannt, der zu berichten weiß, dass schon bald nach der 
Schöpfung die Menschen sich gegen den Sonnengott Re auflehnten. Dieser entsandte daraufhin sein «feuriges Auge», die Göttin Hathor, um die Menschen zu vernichten. Sie befolgte diesen Befehl so gründlich, dass Re ihr Einhalt gebieten musste, um die Menschheit vor der totalen Ausrottung zu retten. Mit Hilfe von 7000 Krügen roten Bieres, die er über das Land ausgießen ließ, täuschte er die Göttin, die daraufhin von den Menschen abließ. Wenn auch dieser Mythos primär einige Riten rund um das ägyptische Hathor-Fest erklären will [11], so schwingt doch mit, dass auch hier das Ausgießen des Blutes (wenn auch symbolisch) im Rahmen eines komplexen Schöpfungsgeschehens Gewalt eindämmt und den Menschen das Leben ermöglicht.

\section{Das kanaanäische Anat-Baal-Epos}

Aus dem kanaanäischen Anat-Baal-Epos, der im ausgehenden 2. Jahrtausend v. Chr. im Bereich Syrien, Libanon und Israel erzählt wurde, kennen wir eine mythische Schilderung, wonach die kriegerische Göttin Anat über die Tötung ihres Geliebten Baal so in Rage gerät, dass sie wie eine Furie in der Welt wütet, bis sie im Blut ihrer Feinde watet und ihre Hände darin wäscht. Sie beruhigt sich erst, nachdem der oberste Gott El ihr in einem Festmahl Brot und Rotwein («Blut des Weinstocks») kredenzt und ihr die Wiedereinsetzung Baals als Gott der Fruchtbarkeit garantiert hat [12].

\section{These: Das Vergießen des Blutes kanalisiert Gewalt und bewirkt Deeskalation.}

Warum das so ist, entzieht sich vorerst noch der Erklärung. Aber ich gehe wohl nicht fehl, wenn ich annehme, dass allein schon der Anblick des vergossenen Bluts als ein Tremendum, ein «Furcht-Erregendes» empfunden wird, das erstarren und einhalten lässt und damit die deeskalierende Reaktion anstößt.

\section{Die ägyptischen Plagen}

Das Alte Testament führt uns in der Erzählung von den ägyptischen Plagen noch in einen weiteren Zusammenhang, der eine nähere Betrachtung verdient und ebenfalls mythische Querbeziehungen aufweist. Hier wird berichtet, dass bereits die erste Plage furchtbaren Schrecken über die Ägypter gebracht hat: Moses nahm den Stab, wie ihm Gott aufgetragen hatte, und schlug vor den Augen des Pharao und seiner Höflinge auf das Wasser des Nil. Da verwandelte sich alles Nilwasser in Blut. Die Fische im Nil starben, und der Nil stank so sehr, dass die Ägypter kein Nilwasser mehr trinken konnten (Exodus 7,19-25).

\section{«Blut-Nil»}

Ich möchte diese biblische Perikope auslegen unter Zuhilfenahme einer ägyptischen Vorstellung. In den medizinischen
Papyri wird immer wieder auf Anubis, den Gott der Einbalsamierung hingewiesen, der in der Medizin die Aufgabe hat, den «Blut-Nil» durch einen Damm aufzuhalten. Gemeint ist damit die Stillung von Blutungen und die Verhinderung von Frühgeburten bei schwangeren Frauen, um so das Leben der Ungeborenen zu retten. Westendorf [13] weist auf einen interessanten Zusammenhang hin: In den «Sprüchen zum Abwehren des Blutes» im Londoner medizinischen Papyrus (BM Nr. 10059) liegt eine Sammlung magischer Zaubersprüche vor, die einen Incubus durch den Wüstengott Seth vorbeugen und eine Fehlgeburt verhindern sollen. Dabei wird ein Ritus beschrieben, in dessen Verlauf ein Amulettknoten in die Vulva eingeführt wird. In diesen Sprüchen wird die Menstruation mit dem Nil verglichen und der Gott Anubis wird aufgerufen, mit einem Damm den «Blut-Nil» aufzuhalten.

Das erinnert an den Mythenkomplex um das Götterpaar Isis und Osiris und seinen Erzfeind, dem Wüstengott Seth. Mit allen Mitteln möchte dieser den Sohn der Isis, den Horusknaben (d.i. der noch ungeborene Pharao) vernichten, um auf diese Weise die Sukzession der ägyptischen Dynastien und Pharaonen abzubrechen, um - so letztlich - die Welt anzuhalten, damit er selbst Herr über das Fruchtland werden kann. Um seinen Angriff auf das Ungeborene zu verhindern, muss der Geburtskanal der Göttin durch ein besonderes Amulett («Tit») geschützt werden, das «Isis-Knoten» oder «Isis-Blut» genannt wird. Gedacht war wohl an einen Schutz-Tampon oder Uterushalsschlaufe. Die entsprechende Hieroglyphe ähnelt dem bekannten Anch-Zeichen. Damit ist die semantische Nähe des «Isis-Bluts» zum Leben graphisch vollzogen und nicht mehr zu übersehen. Blut - Fruchtbarkeit - Leben, eine semantische Symbiose.

Das Fließen des «Blut-Nils» als erste Plage entspricht damit genau der zehnten Plage, der Tötung der ägyptischen Erstgeburt. Uneingedämmter «Blut-Nil» ist unaufhörlich verrinnendes Leben und in der Wirkung identisch mit der Tötung der Leibesfrucht oder der Erstgeborenen. In der Bibel meinen beide Plagen - der «Blut-Nil» und die Tötung der Erstgeburt - dasselbe in doppelter Explikation: Ägypten hat keine Lebensmöglichkeit mehr, wenn es die Hebräer nicht aus der Knechtschaft entlässt.

\section{Inanna und der Gärtner}

Einer der ältesten uns überlieferten Mythen aus dem Zweistromland mit Namen «Inanna und der Gärtner» entstammt der Kultur der Sumerer und dürfte in das 3. Jahrtausend v. Chr. zurückreichen. Er erzählt von der Göttin Inanna, die sich nach langer Reise in einem schönen Garten zur Ruhe legt. In der Nacht missbraucht der Gärtner die Göttin. Auf der Suche nach dem Missetäter füllte sie alle Brunnen des Landes mit Blut, so dass die Menschen nur noch Blut schöpfen konnten. Die Rache der Göttin bedrohte das Leben aller Menschen. Aufgrund des fragmentarischen Charakters des Mythos erfahren wir nicht den Ausgang, aber wichtig ist hier die Parallelität zur ersten ägyptischen Plage im Alten Testament. 


\section{Adonis-Mythos}

Im Bereich des Libanon hat sich der Adonis-Mythos entwickelt. Das Wasser des Nahr Ibrahim (nördlich von Beirut) entspringt in der Grotte von Afqa und färbt sich jedes Frühjahr rot vom Blut des Adonis, der hier bei der Jagd tödlich verletzt wurde. Aus seinen Blutstropfen entstanden die roten Anemonen. Trotz der Motivnähe zum «Blut-Nil» muss dieser Mythos mit dem bereits genannten kanaanäischen Anat-BaalMythos verglichen werden, da es auch ihm um das Erwachen und Sterben der Natur im Rhythmus der Jahreszeiten geht.

\section{Die eucharistische Formel im Neuen Testament}

Nach dem Abschreiten des Alten Testaments ist nun ein Blick in das Neue Testament, den zweiten Teil der christlichen Bibel, notwendig. Drei Aspekte scheinen mir wichtig zu sein: Im Neuen Testament stoßen wir unmittelbar auf die wohl dichteste Aussage der Heiligen Schrift im Bezug auf das Blut. Ausgangspunkt ist das als so genanntes Abendmahl im Neuen Testament beschriebene Herrenmahl Jesu mit seinen Jüngern (Markus-Evangelium 14,22-24 parr.; 1. Korintherbrief 11, 23-27; vgl. Johannes-Evangelium 6,53-56), in dessen Verlauf Brot und Wein genossen werden, Speisen, die von Jesus als sein Leib und als sein Blut den Mahlteilnehmern gereicht werden. Dabei handelt es sich aber nicht um einen einmaligen Vorgang, vielmehr wird durch die Stiftungsformel «Tut dies, sooft ihr es tut, zu meinem Gedächtnis» das Sakrament der Eucharistie der Kirche gestiftet. Natürlich geht es hier nicht um Anthropophagie und Kannibalismus, sondern Jesus Christus bringt sich bleibend in die Gegenwart der Kirche ein und ist - so will es der Glaube - real präsent. Fleisch und Blut stehen für Christus, der sich ganz in diese bleibende Gemeinschaft hineingegeben hat (Johannes-Evangelium 6,53 ff.).

Das Blut des Gekreuzigten wird - so sagt der Text - zur Sühne für uns und unsere Sünden vergossen (Hebräerbrief 9,11-22). Hier fließen die Vorstellungen vom alttestamentlichen Opferlamm, dessen Blut zur Sühne geopfert wird, wie auch die des Sündenbocks, des Paschalammes und die alter, mit Blut geschlossener Bundesrituale zusammen. Hinter dem uns heute weitgehend fremd gewordenen Begriff der «Sühne» steht die Vorstellung, dass jedes Vergehen Gewalt und Zerstörung beinhaltet. Seit alters ist die Menschheit überzeugt, dass solches nicht einfach nur worthaft (z.B. durch Vergebung) wieder ausgeglichen und geheilt werden kann. Im menschlichen Bereich lässt sich eine solche Heilung entweder durch Blutrache oder durch Ersatzleistungen vollbringen, im Bereich Mensch Gott übernimmt das Opferblut des Gekreuzigten diese Aufgabe (Epheserbrief 1,7; Kolosserbrief 1,14). Dieses Blut ist insofern heilend und heilsbedeutsam, als es die durch die Schuld zerbrochene Gottesbeziehung wiederherstellt, den Menschen untereinander und mit Gott versöhnt und den Weg zum Heil frei macht.

Die Kreuzespassion schildern die Evangelisten erstaunlich re- alistisch, um eine metaphorische Deutung auszuschließen. Dieser Realismus zeigt sich z.B. in der beobachteten Fraktionierung des Blutes des Gekreuzigten in «Blut und Wasser» (Johannes-Evangelium 19,34). Dahinter steht ganz sicher lediglich die pure optische Wahrnehmung, nicht jedoch eine prärationale Bluttheorie. Aus dieser Beobachtung hat die Kirche die beiden Sakramente der Eucharistie (ebd. 6,53 ff.) und der Taufe (ebd. 3,5; 13,2-11) begründet.

\section{These: Für die christlichen Kirchen ist das vergossene Blut Christi das entscheidende «Heilstherapeutikum».}

Wenn nun das Neue Testament eine solch dichte Vorstellung vom Blut Christi als die die Welt heilende Arznei hat, dann kann es sich dabei auch auf Vorstellungen vom Blut stützen, die aus der griechischen Welt in die Vorstellungen der Evangelisten eingedrungen waren.

\section{Das Blut in der Kulturgeschichte}

\section{Blut als Sitz des Lebens bei den Griechen}

Spätestens seit dem nachbiblischen Judentum galt das Blut als Sitz der ruach und des pneuma (Buch der Jubiläen 6,7) und als Substanz der Lebenskraft [14]. Das ist so neu nicht, hatte doch schon der Grieche Empedokles im 5. Jahrhundert v. Chr. das Blut als Sitz der Denkkraft angesehen [15]. Entsprechend seiner Lehre von den vier Elementen hat die spätere Humoralpathologie die Lehre von den vier Körperflüssigkeiten Blut, Schleim, helle und dunkle Galle entwickelt. Galen von Pergamon (130-199 n. Chr.) entwickelte die Lehre von den drei Pneumata: spiritus naturalis (venös); spiritus vitalis (arteriell) und spiritus animalis (neural), was im Vergleich zum viel späteren Willliam Harvey (1628) schon eine beachtliche Differenzierung darstellt.

Das Alte Testament wusste um die enge Nähe von Blut und Seele. Kam es hier und in den sonstigen Kulturen lediglich zu Parallelisierungen der Wörter für «Blut» und «Leben», so wird im Koran die alttestamentliche Vorstellung präzisiert und das «Blut» direkt als nafs «Lebensodem» bezeichnet. Blut und Leben werden damit identifiziert!

Ovid [16] eröffnete in seinen Metamorphosen - geschrieben zirka $1 \mathrm{n}$. Chr. - eine bemerkenswerte Perspektive, die weitestgehend aus dem Bewusstsein verschwunden ist: Er erzählt, dass Medea den alten Greis Aeson, den Vater des Jason, durch einen Austausch des Bluts in einen Jüngling verwandelte. Die rabiate Schilderung lässt Medea die Gurgel des Aeson aufschlitzen, um das verjährte Blut ausfließen und ein aufbereitetes Schafsblut einfließen zu lassen. «Weit entflieht die Magerkeit; Blässe und Altersschwäche verschwinden» und nach einer anderen Übersetzung: «voll von erneuertem Blut sind gedrängt die gehöhleten Adern». Dass Francis Potter um 1650 sich von dieser Schilderung zur Bluttransfusion inspirieren ließ, ist beachtlich [17], aber wirklich wichtig ist, dass mit 
dem Blut hier offensichtlich eine Individualisierung des Menschen verbunden wird. Blut ist etwas, was zum Individuum spezifisch hinzugehört, was das Individuum ausmacht, was seinen Charakter prägt. In China pflegte man das Blut der getöteten Feinde zu trinken, um sich ihre Kraft anzueignen. Die Germanen tranken aus demselben Grund das Blut von Bären und Wölfen. Ein Austausch des Bluts verändert das Individuum. Damit aber ist jeder Umgang mit Blut ethisch qualifiziert, wie auch jeder Umgang mit der Eizelle eines Menschen ethisch qualifiziert ist.

\section{Die mechanistische Abwertung des Bluts}

Spätestens seit Descartes ist die Wertschätzung des Bluts ins Gegenteil umgeschlagen. Er hat aus dem mikro- und makrokosmisch verstandenen Blutkreislauf Harveys einen rein mechanistischen Vorgang gemacht, wobei das Blut zu einem Stoff degradiert wurde, der nach mechanischen Gesetzen durch die Körpermaschine geleitet wird [18]. Das Blut hatte aufgehört, Sitz der Seele und des Lebens zu sein. Der niederländische Physiologe Jakob Moleschott (1822-1893) sah das Blut rein materialistisch in Stoffwechselprozesse eingebunden, was wiederum Ludwig Feuerbach zu seiner berühmten Hypothese des «Kartoffelblutes» der Iren führte, die im Freiheitskampf gegen die Engländer keine Chance haben könnten, da diese blutige Steaks essen: «Der Mensch ist, was er isst» [19]. Selbst in dieser rein mechanistischen Sicht schimmert noch durch, dass das Blut als mit individualisierender Kraft ausgestattet gedacht wurde.

4. These: Die Kulturgeschichte wusste noch um die individualisierende Potenz des Blutes. Diese muss wieder neu entdeckt werden, um eine Ethik der Hämotherapie zu entwickeln.

\section{Das Blut in der antiken Mythologie}

Auch ohne direkte Bezüge zur Bibel eröffnet uns die antike Mythologie ein umfangreiches und vielschichtiges «Blutbild». Nun sollte man sich davor hüten, im Nachgang zu Bultmanns Entmythologisierung der Bibel den Mythos als unwissenschaftlich abzutun. Für uns Heutige ist es selbstverständlich, dass die materielle Seite der Wirklichkeit vornehmlich in den Naturwissenschaften, die ideelle vornehmlich in den Geisteswissenschaften behandelt wird. Dieser Unterschied existiert für den Mythos nicht. Alles wissenschaftlich rein Materielle ist für den Mythos zugleich durch etwas Ideelles, nämlich durch ein numinoses Wesen bestimmt, sei es, dass es darin anwesend ist, sei es, dass es dessen Personifikation darstellt. Der «Mythos» ist damit eine hoch qualifizierte Form der Welterklärung aus der vorwissenschaftlichen Phase menschlicher Kultur, besitzt aber eine der Wissenschaft durchaus ebenbürtige, wenn auch verschiedene Rationalität.

\section{Allgemein}

Um ein wichtiges Ergebnis meiner Untersuchungen vorwegzunehmen:

\section{These: Die Religionsgeschichte ist geprägt von der Ambivalenz des Blutes: Fascinosum versus Tremendum. \\ Der Umgang mit Blut heilt und rettet Leben, zugleich kann er den Tod bringen.}

Kennzeichnend ist einerseits die allenthalben $\mathrm{zu}$ beobachtende Scheu vor dem Blut, die schon in prähistorischer Zeit eine Reihe von Tabus entstehen ließ. Andererseits aber erhoffte man sich, durch spezielle Riten die dem Blut innewohnenden Kräfte dienstbar machen zu können. Der ambivalente Charakter dieser Materie, deren Beziehung zum Leben immer schon bekannt war, flößte Ehrfurcht ein. Diese Ambivalenz führte zu einer Polarität in den Vorschriften für den Umgang mit Blut: einerseits war Blutvergießen zu vermeiden, andererseits waren Schlachtopfer (auch Menschenopfer) geboten; einerseits war die Berührung von Blut untersagt, andererseits waren Blutapplikationen geboten. Einerseits machte die Berührung mit Blut unrein, andererseits bewirkte die Applikation mit Blut Heiligung und klerikale Auslese.

Daraus wurden zwei Folgerungen gezogen: Blutvergießen ist zu unterlassen, da es zum Tod führt; Blutzufuhr in Form einer Applikation, eines Getränkes oder eines Trankopfers könne dagegen das Leben mehren und kräftigen. Blut ist gut und Leben spendend, zugleich kann es schlecht und todbringend sein. Hämotherapie heilt und rettet Leben, zugleich kann sie den Menschen töten. Diese Ambivalenz signalisierte schon in der Antike den unbedingten Regelungsbedarf des Umganges mit dem Blut. Das ist heute nicht anders.

\section{Blut in den Schöpfungsmythen}

\section{Altbabylonischer Weltschöpfungsmythos}

Außerhalb des Alten Testamentes begegnet man dem Blut als wesentlichem Bestandteil in allen antiken Schöpfungsmythen. Nach dem altbabylonischen Weltschöpfungsgedicht Enuma Elisch wurden die Menschen aus dem Blut des erschlagenen Gottes Enlil erschaffen. Im Blut (damu) existierte seitdem der göttliche Ursprung des Menschen weiter und äußerte sich in seiner Lebendigkeit. Diese Vorstellung wurde am Alten Testament vorbei vom Islam aufgenommen, wenn es im Koran heißt, die Erschaffung des Menschen sei aus einem Blutklumpen («Embryo») geschehen (Sure 96,2). Die gemeinsame Blutsherkunft machte alle Menschen miteinander verwandt, allerdings in unterschiedlichen Graden. Brüder waren demnach vom selben Blut. Mit «entfernteren» Menschen ließ sich die Brüderschaft durch Blutsverbrüderung herstellen. 
6. These: Schon die antiken Mythenkreise sehen im Blut die entscheidende Verbindung des Geschöpfs Mensch zum Schöpfer Gott. Schöpfung und Tod werden unmittelbar und ursächlich mit dem Blut verbunden.

\section{Mythologie der alten Ägypter}

Nach der Mythologie der alten Ägypter sah man im göttlichen Blut wie in allen göttlichen Ausscheidungen eine schöpferische Kraft. So entstand aus dem Beschneidungsblut des Sonnengottes Re das Götterpaar Hu und Sia, aus dem Nasenbluten des Erdgottes Geb die harzreiche Zeder, aus dem Blut des Bata der Persea-Baum. Trotzdem wurde in Ägypten das Blut allgemein gering geschätzt, so dass in dieser Kultur die Schöpfung der Menschen nicht mit Götterblut in Verbindung gebracht wurde.

\section{Blut in den Vegetationsmythen}

Die schon bei der Analyse der Kain-Abel- und der PlagenGeschichte beigezogenen altorientalischen Mythen haben dem Wesen des Mythos entsprechend - eine Welt erklärende Funktion. Das Waten der Göttinnen Hathor und Anat im Blut bewirkt zwar einen sprunghaften Rückgang des Aggressionspotentials, hat aber im Gesamtbild des jeweiligen Mythos eine übergeordnete Aufgabe: Die hier zu beobachtenden Handlungsabfolgen stehen für den alljährlich sich abspielenden Vegetationszyklus - hier besonders für die Überwindung der spätsommerlichen Dürre durch den fruchtbringenden Winterregen. Warum aber gebraucht der Mythos ein solch mächtiges Bild? Der Grund liegt darin, dass die unbekannten Mächte hinter dem Vegetationszyklus nur mit mächtigen Metaphern erklärt werden konnten. Der dynamische und ununterbrochene Vegetationszyklus ist lebenswichtig für die bäuerliche $\mathrm{Be}$ völkerung Kanaans, eines Gebietes, das Westsyrien, Libanon und Israel umfasste. Deshalb musste man alle rituellen Kräfte des Kults darauf verwenden, diesen Vorgang zu stabilisieren. Das ließ sich aber nur mittels des Rituals bewerkstelligen.

\section{Blut im antiken Ritual}

\section{Blut in apotropäischen (abwehrenden) Riten}

Aus allen antiken Kulturen ist uns ein ritueller Umgang mit Blut bekannt, der sich häufig nicht eindeutig von medizinischen Applikationen unterscheiden lässt. Die sprichwörtliche Scheu vor dem Blut ist das Hauptmerkmal in den vielen ethnographischen und religionsgeschichtlichen Belegen. Vom Blut gingen viele apotropäische, volksmedizinische und sakramentale Wirkungen aus, die man durch Magie zu beherrschen suchte. Da Blut als krafthaltig galt, war es ein ausgezeichnetes Mittel zur Abwehr von Dämonen. Dem diente z.B. in Rom das Bestreichen der Grenzsteine mit Blut an den Terminalien (23. Februar) oder der Türpfosten zur Abwehr von Schadenzauber.
Im alten Israel applizierte Zippora in der rätselhaften Notiz vom «Blutbräutigam» (Exodus 4,25 f.) das Beschneidungsblut auf die Beine des jungen Moses und rettete ihm damit das Leben, denn der Dämon drehte ab. Die Hebräer bestrichen die Türpfosten und Oberschwellen mit Blut zur Abwehr des Würgengels (Exodus 12,13-23). Dahinter steht ein alter Nomadenbrauch, der die Herde vor dämonischen Einwirkungen schützen soll [20]. Die Beduinen kennen mehrere Anlässe, die Türpfosten mit Blut zu bestreichen: zum Zeichen eines eingelösten Gelübdes oder wenn die Vorbewohner eines Hauses starben oder wenn ein Kind an der Türschwelle gestürzt war. Nach Plinius kannten auch die Römer die Sitte, die Türpfosten mit einer Mischung aus Hyänenblut und Polenta gegen die magorum artes zu bestreichen. Durch zunehmende Vergeistigung und Rationalisierung der israelitischen Religion wurde diese dämonische Komponente verdrängt: von nun an dienten solche Bräuche lediglich der Erinnerung, wenn z.B. die Israeliten aufgefordert wurden, die Tora, das Gesetz Gottes, auf die Türpfosten zu schreiben (Deuteronomium 6,9).

\section{Blut in Bundesriten}

Eine völlig andere Vorstellung steht hinter dem Blutbund z.B. im Kontext der Verschwörung Catilinas in Rom (62 v. Chr.). Da das Blut Träger des individuellen Lebens und essentieller Bestandteil des Spenders ist, gibt sich der einen solchen Bund Schließende selbst in diesen Bund ein und besiegelt dessen Unauflösbarkeit. Es verwundert nicht, dass sich dieses alte prähistorische Koalitionsmuster bis heute gehalten hat, da es eine hohe Kohäsionskraft in sich birgt. So manches Mädchen wird im Mittelalter einen Liebestrank - mit einem Tropfen eigenen (Menstruations-)Blutes - gebraut haben, um ihren Liebsten an sich zu binden. Dabei tat es nichts anderes als schon der altägyptische Gott Osiris, der mit einem aus seinem Blut und Wein gemischten Getränk die Göttin Isis an sich zu binden suchte [21]. Fügte man dem Trank gar einen Tropfen Karnickel- oder Hasenblut bei, dann versprach man sich eine reichliche Vermehrung.

Im Blut ist also etwas vom Wesen der jeweiligen Person enthalten. Zusätzlich hat es eine Gemeinschaft stiftende Funktion, die zur rituellen Verwendung besonders im Bereich von Initiationen führt. In den Attis-Mysterien und im MithrasKult wurden die Initianden mit Stier- oder Widderblut übergossen. Sie wurden durch diesen Ritus mit der Kraft des getöteten Tiers ausgestattet, zum Mysten geweiht und eines höheren Lebens teilhaftig [6, 22].

\section{Blut als Arznei}

Man sah allenthalben im Blut die Lebenskraft situiert. Deshalb fing man seit der Römerzeit bis ins frühe 19. Jahrhundert gegen behördliche Verbote das Blut der Hingerichteten auf, um es als Heilmittel, besonders gegen Epilepsie, zu verwenden.

Auch wenn die alten Ägypter mit dem Blut rituell wenig anfangen konnten, da sie es wie Eiter, Schweiß und Urin als ein 
Krankheitssekret deuteten, so konnten sie es doch gelegentlich als Arznei einsetzen. Das Verbleiben des Bluts im Leib der Schwangeren war für den Embryo lebenswichtig und musste deshalb durch Amulette und Zauber geschützt werden. Als Menstruationsblut war es zwar unrein, aber die Applikation mit Menstruationsblut sollte ein allzu üppiges Wachstum der Brüste verhindern. Diarrhö behandelte man mit Rinderoder Schweineblut.

Im Mittelalter behandelte man Augenkrankheiten mit Blut vom Rebhuhn, Mandelentzündung mit Schwalbenblut und die Schwindsucht mit Schlangenblut. Dieser Katalog kann beliebig fortgesetzt werden. Bekanntlich machte das Baden im Drachenblut unverwundbar (Siegfried-Sage).

\section{Ethische Implikationen - ein Versuch}

Seit alters sind Ethik und Religion eng miteinander verbunden. Der Religion geht es - wie der Begriff schon sagt - um eine Rückbindung (re-ligio) des Menschen an einen Gott, aus der er Verhaltensregeln gewinnt und ableitet. Sittlichkeit und Religion bilden eine Einheit, die dem Gelingen des menschlichen Zusammenlebens dienen soll. Aus den Mythen leitet der Mensch Muster für die Deutung der Welt und für die Regelung seines Zusammenlebens ab. In der Bibel ist es im Prinzip genau so, nur kommt hier - wie wir glauben - die Offenbarung durch Gott hinzu.

Im Unterschied zu den altorientalischen Mythen sah das Alte Testament «Blut» und «Leben» eng beieinander (Levitikus 17,11,14; Deuteronomium 12,23). Dabei konnte «Blut» wie eine dem Menschen innewohnende selbständige Wirkmacht verstanden werden. Das Blut des erschlagenen Abel dringt in das Erdreich ein («der Ackerboden hat seinen Mund aufgesperrt, um das Blut deines Bruders aufzunehmen») und aus dem Erdreich heraus schreit das Blut des Erschlagenen zu Gott (Genesis 4,10). Dahinter steht eine animistische Vorstellung, die offensichtlich noch aus der prähistorischen Zeit stammt, zugleich aber auch eine noch primitive Ahnung darum, dass im Blut das Wesen des Menschen, der Mensch selbst anwesend ist.

\section{Blutvergießen}

Der größte Frevel im Alten Testament ist das Vergießen des Blutes (Genesis 9,6), das bereits nach der ältesten Rechtsnormen-Sammlung in den so genannten «noachitischen Geboten» (Gebote an Noach nach der Sintflut) durch das Blut des Täters gesühnt werden muss. Überhaupt wird Gott für jedes vergossene Blut - sei es Tier, sei es Mensch - Rechenschaft einfordern (ebd. V. 5), denn hier geht es - wie der Text überraschend sagt - um eine Verletzung der Gottesebenbildlichkeit des Menschen. Diese - sicherlich sekundär angefügte Begründung ist höchst interessant, will sie doch das Gebot mit allerhöchster Autorität ausstatten. Innerbiblisch ist diese mächtige theologische Begründung nicht zu erklären, denn im
Zusammenhang mit der Schöpfung und der Gottesebenbildlichkeit taucht das Blut niemals auf. Erst ein Blick in die mesopotamische Nachbarkultur weist auf den mythischen Hintergrund: dort nämlich sind die Menschen aus dem Blut des erschlagenen Gottes Enlil entstanden. Das Blut also ist der Träger der göttlichen Herkunft des Menschen! Daraus entwickelt sich folgerichtig die

\section{These: Jedes Blutvergießen ist grundsätzlich ein Eingriff in die Hoheitsrechte Gottes.}

\section{Blutapplikationen zur Reinigung und Weihe}

Es ist und bleibt eine Aufgabe der religionsgeschichtlichen Forschung aufzudecken, wie sich der Umschwung von der Wertung des Bluts als eines geradezu göttlichen Elements zur Reinigung und Weihung hin zu einer Größe, deren Kontamination wie eine Berührung von Leichen und Aas den Menschen unrein macht und umfangreiche Reinigungsriten erfordert, vollzogen hat.

Bei der Priesterweihe wurde zur Reinigung und Entsündigung das Blut des Opfertiers an Ohrläppchen, große Zehen und Daumen des $\mathrm{zu}$ weihenden Priesters appliziert (Exodus 29,20). Auch das Volk wurde im Zusammenhang einer Bundesschluss-Feier mit dem Blut des Opfertiers besprengt (ebd. 24,5 ff.). Im Grunde genommen ging solches Tun auf einen uralten Vertragsfluch zurück, wonach der Bundesbrüchige mit dem Tod bestraft werden sollte. Ihm würde es so ergehen, wie es dem Opfertier ergangen ist.

\section{Verbot des Blutgenusses}

Im Alten Testament war das Blut ohne Zweifel ein echtes Reservat des Gottes Israels. Blut durfte nicht und niemals konsumiert werden (Levitikus 11,3 f; 17,10-14). Bei der Schlachtung musste man das Blut ausfließen lassen und es nachher mit Erde bedecken (ebd. 17,12 f). Um der Gottheit das Blut eines Opfertiers zurückzuerstatten, goss man es auf den Altar (Richterbuch 6,20) oder an den Altarfuß.

Da Blut als Leben verstanden wurde, implizierte der Verzicht auf den Blutgenuss - unabhängig von möglichen latenten Tabus aus der Prähistorie - eine theologisch-anthropologische Komponente, nämlich den Verzicht auf eine schrankenlose Herrschaft und Aneignung des Lebens. «Das Blut wird dem Schöpfergott als dem einzigen und absoluten Herrn über Leben und Tod zurückerstattet und das menschliche Geschöpf erweist dem Geheimnis des Lebens durch Selbstbescheidung seine Reverenz» [23]. Insofern kann die Rückgabe des Bluts an den Schöpfergott als Zeichen des Dankes für die Erlaubnis der Tiertötung und Nahrungsbeschaffung gesehen werden. Auch das Neue Testament hat diese Vorschriften übernommen: Bluttat (Matthäus-Evangelium 23,35) und Blutgenuss (Apostelgeschichte 15,29) galten als Kapitaldelikte. Obwohl das Apostelkonzil den Heidenchristen gegenüber Konzessionen machen musste (ebd. Kap. 15), blieb der Kern der Bestimmungen erhalten und wurde in den so genannten Jako- 
bus-Klauseln festgelegt: auch den Heidenchristen war es verboten, Götzenopferfleisch, unausgeblutetes Fleisch und Blut zu verzehren (ebd. 20,29). Die so genannte «westliche Texttradition» hat verhindert, dass daraus ein allgemeines Speisegebot abgeleitet worden ist; es handelt sich lediglich um ein Verbot des Blutvergießens.

\section{Blut und Opfer}

Die im Zusammenhang der alttestamentlichen Opferpraxis durchgeführten Blutmanipulationen waren von einer solch tief greifenden Ehrfurcht geprägt, dass sie durch ein subtiles Regelwerk geschützt werden mussten, um jeden rituellen weil schädlichen - Fehler zu vermeiden. Ein solches Regelwerk findet sich in Levitikus $1 \mathrm{f}$ : je nach Opferart musste das Blut des Opfertiers vor dem Vorhang des Heiligtums versprengt werden, oder es musste ringsum an den Altar gesprengt werden, oder die Hörner des Altares sollten damit bestrichen werden. Am Versöhnungstag erreichte die Blutmanipulation ihre höchste Steigerung, wenn der Altar, seine Hörner und zusätzlich die Deckplatte der Bundeslade siebenmal mit Blut besprengt wurden (Levitikus 16,14 ff). Angesichts dieser unschwer als fortdauernde Magie erkennbaren Rituale gerät der Exeget in enorme Deutungsschwierigkeiten. Obwohl Magie im Alten Testament verboten war, wurden diese Handlungen jedoch von Gott selbst angeordnet. Der Bibeltext selbst gibt uns eine Deutungshilfe, wenn es dort heißt, dass «das Blut an den Altar gegeben werde, damit es Sühne für euch schaffe, denn das Blut schafft durch das Leben darin Sühne» (ebd. 17,11).

Man muss wissen, dass bereits im Judentum selbst die Sitte der blutigen Opfer aufgegeben wurde. Bereits die Gemeinde von Qumran am Toten Meer (ab zirka 100 v. Chr.) spiritualisierte die Blutriten und ersetzte sie durch Gebete, Gesetzeswerk und rechten Lebenswandel. Die jüdische Synagoge begründete später das Aufgeben der Blutriten mit der Zerstörung des Tempels durch die Römer 70 n. Chr. Zum christlichen Opferbegriff muss an dieser Stelle ein Literaturhinweis genügen [24].

\section{Ethische Vorbehalte gegen die Bluttransfusion}

Meine abschließenden Betrachtungen der gegenwärtig bekannten ethisch-religiös motivierten Vorbehalte gegen die Bluttransfusion möchte ich lediglich auf die Zeugen Jehovas beschränken. Auch auf die Gefahr hin, die umfassende Argumentation nicht ausreichend oder gar erschöpfend würdigen zu können, möchte ich doch die Hauptpunkte der Argumentation kurz vorstellen. Die Ansicht der Zeugen Jehovas [25], Organtransplantationen sowie Transfusionen von Albumin, Immunglobulinen, Faktoren zur Blutgerinnung und Blutersatzstoffen zu akzeptieren, aber Bluttransfusionen selbst nicht, erscheint auf dem ersten Blick konfus und unüberlegt; dies umso mehr, da doch der Einsatz von Dialyse- und Herz-
Lungen-Maschinen akzeptiert wird. Die Einschränkung, die bei letzterem gemacht wird, führt zur inneren Logik der Argumentation: bei solchen Verfahren darf kein Blut außerhalb des Körpers aufbewahrt oder zwischengelagert werden und der Blutkreislauf darf außerhalb des Körpers nicht unterbrochen werden.

Die Zeugen Jehovas argumentieren auf der Basis der eben vorgestellten Bibelstellen, die allesamt den verbotenen Blutgenuss zum Gegenstand haben: Genesis 9,3 f; Levitikus 17, 13,14; Deuteronomium 12,23,25; Apostelgeschichte 15,20,29. Obwohl diese alttestamentlichen Speiseverbote unter anderem im Matthäus-Evangelium 15,11; in Apostelgeschichte 10,15; im Römerbrief 14,14 und im 1. Korintherbrief 10, 23-11,1 aufgehoben sind, werden sie angeführt. Nach Meinung der christlichen und auch weiter Bereiche der jüdischen Bibelwissenschaft hat die Heranziehung biblischer Texte nur in einem historisch-kritischen Zugriff zu geschehen, die Zeugen Jehovas argumentieren hingegen fundamentalistisch [26]. Dagegen ist einzuwenden, dass alle angeführten Bibelstellen Speisegebote oder Speiseverbote sind, den Sachverhalt einer Bluttransfusion wesentlich nicht betreffen. Die Weisungen der Zeugen Jehovas berufen sich auf göttliche Verbote, die es so nicht gibt, sind also unbiblisch; sie missachten die biblischen Toleranzbestimmungen des Neuen Testaments, sind also unchristlich; sie lassen lebensrettende Maßnahmen nicht zu, sind also unmenschlich und wider den Gott gerichtet, der das Leben will!

\section{Schlussbemerkungen}

Sowohl Mythos wie Ethik des Umgangs mit Blut waren geprägt von einer durchgehend zu beobachtenden allerhöchsten Wertschätzung dieser ambivalenten Materie. Schon der sprachliche Befund zeigte uns eine ehrfurchtsvolle Distanz zum Gemeinten, wenn konsequent der Begriff vermieden und umschrieben wurde. Bis in die Frühantike zurück reicht das Wissen darum, dass das Vergießen des Bluts Gewalt kanalisieren und Deeskalation bewirken kann.

Die Religionsgeschichte war und ist geprägt von der Ambivalenz des Bluts: Fascinosum versus Tremendum. Der Umgang mit Blut kann heilen und Leben retten, zugleich kann er auch den Tod bringen. Diese Ambivalenz führte zur Reflexion in den antiken Mythenkreisen, die im Blut die entscheidende Verbindung des Geschöpfs Mensch zum Schöpfergott sahen. Daraus entwickelte das Alte Testament die komplizierte Vorstellung von der Gottesebenbildlichkeit des Menschen. Allein schon die Notwendigkeit eines äußerst sorgsamen Umgangs mit dem Blut als alleinigem göttlichen Reservat - jedes Blutvergießen ist demnach grundsätzlich ein Eingriff in die Hoheitsrechte Gottes - führte zur vollständigen Delegation dieses Umgangs an die Priester und entzog ihn völlig dem profanen Bereich. Das muss uns lehren, dass von hier her die Arbeit der Hämatologen und Hämotherapeuten von äußerster 
Sorgfalt, Präzision und geradezu rituellem Wissen bestimmt sein muss. Wie das alttestamentliche Ritual nur im Zustand und im Bereich der rituellen Reinheit gelingen konnte, so bleiben Reinheit und Sorgfalt oberstes Gebot im Umgang mit Blut.

\section{These: Das Blut ist ein Reservat Gottes. Darin gründet die ethische Pflicht zu Verantwortung und Sorgfalt.}

Die Kulturgeschichte wusste noch um die individualisierende Potenz des Bluts. Diese muss wieder neu entdeckt werden, um eine Ethik der Hämotherapie zu entwickeln. Der Blutspender gibt sich und von sich, der Patient nimmt ihn und von ihm. Das soll gar nicht pathetisch ausgezogen werden, aber hier ist eine Wirklichkeit angesprochen, die weitgehend unserer Wahrnehmung entschwunden ist.

Jede medizinische Ethik, die sich auf Achtung und Erhalt des Lebens ausrichtet, muss erst recht eine Ethik sein, die den Umgang mit Blut betrifft, denn wie in kaum einer anderen medizinischen Disziplin verdichtet sich im Umgang mit dem Blut der Umgang mit dem Leben. Nimmt der Transfusionsmediziner Blut vom Menschen, so hat er vom Leben genommen. Und seine Aufgabe ist es, dieses dem Leben wieder zuzuführen, um neues Leben zu ermöglichen - eine Partizipation an Gottes Schöpferhandeln. Sein Tun ist im theologischen Sinne die Fortführung der Schöpfung als creatio prima in eine dauerhafte Schöpfungserhaltung, die creatio continua. So wie der Schöpfer das Leben will, so sind die Hämotherapeuten dem Leben verpflichtet. Ihre Verantwortung besteht darin, alles - aber auch alles - zu tun, was die Leben spendende Funktion des Bluts ermöglicht, und alles - aber auch alles - zu vermeiden, was die Tod ermöglichende Ambivalenz des Bluts aktivieren könnte.

Wir alle sind in der Hand Gottes, Transfusionsmediziner aber sind in gewisser Weise diese Hand Gottes, die Heilung wirken will und wirken kann, und sollten für sich diese wichtige Aufgabe der Mitarbeit an Gottes Schöpfung akzeptieren und daraus Freude für ihre Berufung gewinnen.

\section{Literatur}

1 Karl Kardinal Lehmann: Chancen und Grenzen der Modernen Medizin. Plenarvortrag beim 14. Jahrestag des Medizinischen Dienstes der Krankenversicherung in Hessen am 31. Januar 2005 in Bad Wildungen. www.bistummainz.de/opencms/ sites/bistum/bistum/kardinal/texte/texte_2005/wildungen.html.

2 Gadebusch Bondio M: Blood in History and Blood Histories, Firenze, Micrologus' Library, 2005.

3 Kluge F: Etymologisches Wörterbuch der deutschen Sprache, 23. Aufl. Berlin, de Gruyter, 1999, p121.

4 Pfeifer W (Hrsg): Etymologisches Wörterbuch des Deutschen, Band I, 2. Aufl. München, dtv, 1989, p 192.

5 Golther W: Handbuch der Germanischen Mythologie. Wiesbaden, Athenaion, 2004, p 668.

6 Wissmann H: Blut I., Religionsgeschichtlich, Theologische Realenzyklopädie VI. Berlin, de Gruyter, 1980, pp 727-729, bes. 727 .

7 Bergman J,. Kedar-Kopfstein B: dam, Theologisches Wörterbuch zum Alten Testament II, Stuttgart, Kohlhammer, 1977, pp 248-266.

8 Moscati S: An Introduction to the Comparative Grammar of the Semitic Languages. Porta Linguarium Orientalium NS 6, Wiesbaden, Harrassowitz 1964, p 73.

9 Koch K: Der Spruch «Sein Blut bleibe auf seinem Haupt» und die israelitische Auffassung vom vergossenen Blut, Vetus Testamentum 1962;12: 396-416, bes. 406 .
10 Schwager R: Brauchen wir einen Sündenbock? Gewalt und Erlösung in den biblischen Schriften. München, Kösel, 1978.

11 Assmann J: Ägypten. Theologie und Frömmigkeit einer frühen Hochkultur, UTb 366, Stuttgart, Kohlhammer, 1991, p 139.

12 KTU 1,3 II 25-30; in Dietrich M, Loretz O (Hrsg): Texte aus der Umwelt des Alten Testaments III. Mythen und Epen IV, Gütersloh, Gütersloher Verlagsanstalt, 1997, pp 1139.

13 Westendorf W: Beiträge aus und zu den medizinischen Texten, II. Das Isisblut-Symbol. ZÄS 1966; 92:144-154.

14 Böcher O: Blut II. Biblische und frühjüdische Auffassungen. Theologische Realenzyklopädie VI. Berlin, de Gruyter, 1980, 729-736, bes. 731 f.

15 Diels H, Kranz W: Die Fragmente der Vorsokratiker. Griechisch und Deutsch von Hermann Diels; in Kranz W (Hrsg): Band 1. Hildesheim, Weidmann, 1974, pp 350 .

16 Ovid: Metamorphosen, Buch VII, 159-293, bes. 285 ff. Stuttgart, Reclam, 2003, p 349.

17 Schicktanz S: Mensch - Tier - Chimären. Bemerkungen zur Transplantationsmedizin und ihrer Geschichte; in Drux R (Hrsg): Der Frankenstein-Komplex. Kulturgeschichtliche Aspekte des Traums vom künstlichen Menschen. Frankfurt/M., Suhrkamp, 1999, pp 184-203, bes. 189.

18 Rotschuh KE: René Descartes und die Theorie der Lebenserscheinungen. Sudhoffs Archiv 1966;50/1: 25-42.
19 Thies E (Hrsg): Ludwig Feuerbach. Werke, Bd. 4 , Kritiken und Abhandlungen III (1844-1866), Frankfurt/M., Suhrkamp, 1975, pp 243-265, bes. 265.

20 Canaan T: Das Blut in den Sitten und im Aberglauben des palästinischen Arabers, ZDPV 1963;79: 8-23, bes. $20 \mathrm{f}$.

21 Lexa F: La Magie dans l'Égypte antique, Bd. 2, Paris, Paul Geuthner, 1925, pp 139-143.

22 Fischer U: Zur Liturgie des Umbandakultes. Eine Untersuchung zu den Kultriten oder Amtshandlungen der synkretistischen Neureligion der Umbanda in Brasilien. Zeitschrift für Religions- und Geistesgeschichte, Beih. 13. Leiden, Brill, 1970, p 25.

23 Baranzke H: Interkulturelle Bioethik - Beispiel Rituelles Schlachten. Einblicke in den Zusammenhang von Leben, Töten und Essen, Zeitschrift für Didaktik der Philosophie und Ethik 2004:241-250, bes. 245 .

24 Brandt S: War Jesu Tod ein «Opfer»? Perspektivenwechsel im Blick auf eine klassische theologische Frage; in Weth R (Hrsg), Das Kreuz Jesu: Gewalt Opfer - Sühne, Neukirchen-Vluyn, Neukirchener Verlag, 2001, pp 64-76.

25 Carbonneau A: Ethical Issues and the Religious and Historical Basis for the Objection of Jehovah's Witnesses to Blood Transfusion Therapy. Lewinston, NY, 2004.

26 Kraus W: Die Biomedizin als Gegenstand des interkulturellen Diskurses; in Schicketanz S, Tannert C, Wiedemann P (Hrsg): Kulturelle Aspekte der Biomedizin. Bioethik, Religionen und Alltagsperspektiven, Kultur der Medizin 9, Frankfurt/M., Campus, 2003, pp 107-131, bes. 109 